\title{
Author Correction: Charge order and superconductivity in kagome materials
}

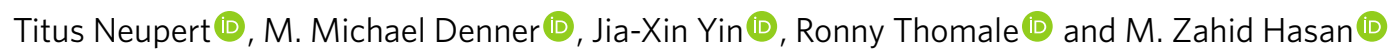

Correction to: Nature Physics https://doi.org/10.1038/s41567-021-01404-y, published online 2 December 2021.

In the version of this Perspective initially published, there was a mistake in the current pattern presented in the right-hand panel of Fig. 2f. The panel has been replaced and the original and revised versions are shown below. Further, the caption for Fig. $2 \mathrm{f}$ has been clarified to read "Arrows indicate the direction of complex hoppings with positive imaginary part (small hopping strength light grey, large hopping strength dark grey) (left) and currents (middle, right) (small magnitude, light grey; large magnitude, dark grey) and background colours indicate positive (red) and negative (blue) fluxes through an area," replacing, in part, "Arrows indicate the direction of complex hoppings with positive imaginary part (small hopping strength light grey, large hopping strength dark grey)...." The changes have been made in the online version of the article.

f

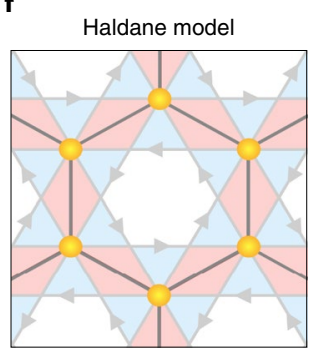

f

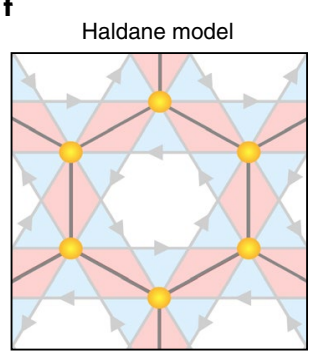

Original

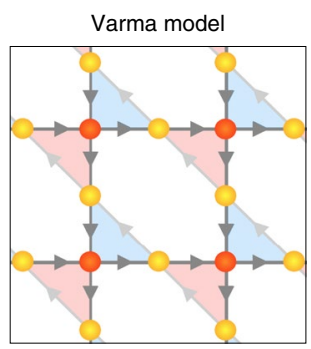

Corrected

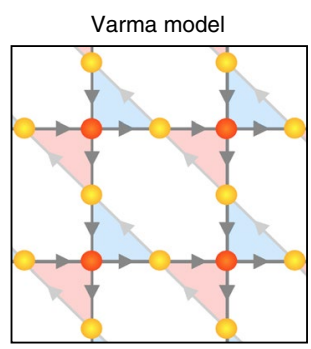

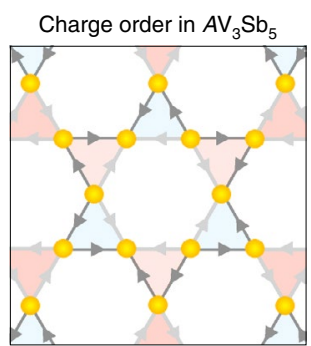

Charge order in $\mathrm{AV}_{3} \mathrm{Sb}_{5}$

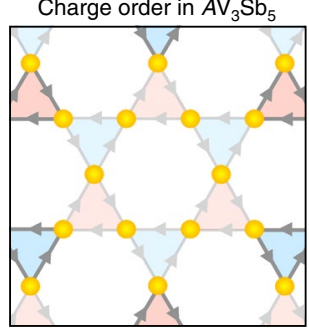

Fig. 2f | Original and corrected.

Published online: 2 February 2022

https://doi.org/10.1038/s41567-022-01528-9

(c) Springer Nature Limited 2022 